tumour very hard, and entirely free from anything like pulsation or bruit; an anastomosing vessel can be felt at the upper part of the tumour, somewhat larger than on the last examination. I may now say, fairly, he is cured, as all that remains is a little stiffness at the knee-joint. It is another triumph to the Dublin men, who originated the plan of cure; for though it had been tried long since, yet the true principle had not been developed, as it now is. I am more satisfied with this case than all the operations I have performed, however successful. Amputation of limbs, hernia, trephining, \&e.,-what are they, compared to the bloodless cure of an important and serious disease? A modification of my compressor might be used even in brachial aneurisms; and $I$ cannot see but that even axillary aneurisms might be reached by it over the sub clavian.

Carlisle, 1851 .

\section{ON THE TREATMENT OF DIABETES.}

By JABEZ HOGG, M.R.C.S., London.

Tre intractable nature of this malady has induced me to devote considerable attention to it, in the hope of being able to devise some plan of treatment upon which we may place more reliance than upon those which are ordinarily adopted, and which, unfortunately, are too often followed by an unsuccessful issue. Those chemical principles which should guide us in the management of this affection $I$ have endeavoured to carry out to a greater extent than $I$ believe has hitherto been attempted; and the happy result of the case I am about to relate will (to say the least) warrant a further trial of the remedy employed.

The singularly surprising phenomenon of the conversion of a large portion of the materials of life into sugar, led me to reflect upon this abnormal condition, and especially so as I found that great uncertainty of action attended all the medicines employed, and but few patients were relieved, and cures were seldom effected.

The chief indications of treatment appears to be the pre. vention of the elaboration of the saccharine poison in (the laboratory of life) the stomach, and to restore the defective power of the digestive apparatus. These ends I sought to fulfil by employing some medicine that would effectually arrest the saccharine fermentation; and I attempted to accomplish my object with a sulphuret of arsenic, administered as I shall detail in the following case which came under my charge.

The chemical difficulty that presented itself to me was the conversion of the sulphuret of arsenic into a soluble sulphuret, which would not accumulate in the system, and become a more than ordinarily dangerous remedy to deal with. 'This I was enabled to effect by combining the hydrosulphuret of ammonia with the liquor arsenicalis.

The first trial I made of this preparation was upon a poor man, J. G., living near Burton-crescent, in a cellar, and otherwise, too, most unfavourably situated for a trial of the remedy. When he applied to me he had suffered about two years from the disease; he had been an inmate of two hospitals, and was discharged unrelieved from the second one about six weeks previous to my seeing him, which was on the 14th December, 1850. He had then been entirely confined to his bed for twelve days; during the last twenty-four hours, and for many days previous, he had passed about three gallons of urine, very sweet to the taste, and of the specific gravity 1:050. Trom mer's test showed the presence of sugar, and upon the addition of yenst I obtained several grains of sugar, the crystals of which I satisfactorily determined under the microscope; pulse smal and qaick, 120 ; cough very troublesome; face œdematous. Ordered a meat diet, with a little toasted bread; and as the thirst was great, tea only, without milk or sugar, with a tablespoonful of the following mixture every four hours :-Liquor arsenicalis, two drachms; hydrosulphuret of ammonia, twenty minims; tincture of henbane, and tincture of lavender, each two drachms; infusion of buchu, eight ounces. To have five grains of soap-and opinm pill at bed-time.

Dec. 16th.-A decided improvement manifest; had passed only his usual quantity of water; specific gravity, 1.045; slept several hours; cough and œdema relieved. Continue mixture and pill at night.

18th.-Improving; pulse 100; not so irritable; urine still decreasing in quantity; specific gravity as before; appetite more natural; cough not so troublesome; had had some comfortable sleep. To continue the medicine.

20th.-Three quarts of urine had been passed during the last twenty-four hours; specific gravity 1.040; cough troublesome, from having neglected to take his pill; bowels confined. Continue mixture, omitting buchu, and adding infusion of calnmba. To have ten grains of aloes-and-myrrh pill at bedtime.

22nd.-Decidedly better in every particular. To continue medicine.

26th.-Improved in appearance, and able to sit up nearly all day; arine, specific gravity 1.035 ; two quarts only passed during twenty-four hours; cough not so troublesome, and expresses himself greatly relieved. To continue the same plan of treatment.

January 2nd, 1851.-Had been living too freely during Christmas; his cough more troublesome, and general health disturbed; but the urine was not affected, nor increased in quantity. To continue the mixture, and take two soap-andopium pills during the next twenty-four hours.

6th.-He was again improving, and I ordered him an additional senna draught to act a little more freely upon the bowels, and to continue his mixture.

10th.- - He was in every respect doing well.

16th.-The urine up to this time had been passed in natura quantities; specific gravity 1.025 , and I conld scarcely satisfy myself of the presence of sugar, his bowels continuing costive. I discontinued the mixture, and ordered him a grain of quinine, with small doses of sulphate of magnesia in infusion of roses three times a day, with the soap pill as before; and up to the 30 th he continued to improve; the cough only remaining to give me any anxiety, he having suffered from a winter cough for many years. I advised him to remove from his underground dwelling to one lighter and more airy. I now felt assured that I had completely arrested the diabetic disease, and sawv no more of my patient for many weeks, when I was again sent for, and found him in this same miserable underground lodging. Thus unfavourably circumstanced, and badly fed, phthisical symptoms set in, and of this disease he died at the end of three months; but without the diabetic state of the urine having reappeared, as I carefully noted its condition from time. I regret being unable to supply the post-morten appearances, the friends most obstinately refusing me permis sion to examine the body.

I have during the year treated another case in a much earlier stage of the disease, and consequently of a milder form, and less complicated than the one above given, with smaller doses of the same mixture, and was gratified to observe the same rapid change. The patient, a boy aged sixteen, was so far recovered at the expiration of a fortnight, as to be enabled to remove to the sca-side: he has had no return of the disease, and is now in perfect health.

Gower-street, Bedford-square, 1851.

\section{ON CHRONIC COLICA PICTONUM.}

\section{Br WILLIAM NORRIS, M.D., \&c., Stourbridge.}

Paintrars, glass-cutters, and persons employed in lead manufactories, it is well known, are often affected by the poison of lead, producing acute painter's colic; but I have yet to learn that the effects of lead have been minutely described as a distinct disease, when it occurs in a more slow and insidious manner, making persons unfit for employment, without assuming the more severe form of the disease.

Acute painter's colic frequently occurs; but I believe that a chronic form of the disease appears ten times more frequently, and goes through its stages almost unnoticed and unknown; and that the health of thousands suffers, and many sustain an irreparable injury to the constitution, because proper and precautionary measures have not been adopted at the onset of the disease.

I have lately attended two very severe cases, in which the patients suffered from frequent pain many weeks, and I candidly confess I was not aware of the nature of the disease till I examined the gums; which points out the necessity of ascertaining the occupation of persons when they apply to us with pain and disorder of the digestive organs.

I will endeavour to give a concise account of this disease, which may run on for years without assuming the acute character: it may be denominated chronic painter's colic, and in my humble judgment ought to be distinctly classed by all systematic writers on medicine.

When persons have been employed several years, and sometimes sooner, the gums become enlarged and flabby, not blue they have often pains in the loins, or some parts of the muscular system, and soon look sallow and thin, with more or less 
disorder in the digestive organs, and sometimes a sweet taste in the throat; these symptoms may go on ten or twenty years before they end in the acute or chronic form of the disease, when the leaden tinge in the gums will be found, proving the system to be now saturated with lead; and if the disease assume a chronic character, the symptoms will be somerhat similar to the acute, in a milder degree-namcly, constipation of the bowels, sometines diarrhoe ; a coppery taste; tongue covered with a dark cream-colonred mucus; a griping pain in the bowels many hours during the day and night, ofteir around the umbilicus, extending over many parts of the abdominal region; loss of appetite, nansea and sometimes vomiting; of ten an exceedingly sweet taste in the throat and faucrs; great depression in the nervous and muscular system; seldon excitement in the vascular system; pulse generally slow and feeblo; the skin dry, and all the sucretions diminished; kidneys torpid; urine scanty, and of a dark colour.

Treatment.-Mild purgatives daily, or every other day; calomel and opium every six or eight hours; then indide of potass in one or two grain doses. If the pain is frecuent and distressing, occasional doses of laudanum. Diarrhoa is sometimes kept up by scybala or vitiated secretions, and may require castor-oil, previons to the nse of astringents. Whe diet should be light and nutritious; dilute freely with mucilaginous drinks. When pain is unusually severe, the warm-bath has a striking effect; bran ponltices and hot salt are always nseful, and may be applied to any of the affected rarts.

When patients have premonitory symptoms, they shonld be removed from their cmployment, the sin frequently washed, and the clothing chenged, with proper remedies; by these precautions, many serious attacks may probably be prevented. The sweet taste from the throat may be removed by frequent gargling with water, or water mixed with iodide of potass

Those who are predisposed to the disease may take iodide of potass once or tivice every year; "and as a preventive take sulphuric acid lemonade." $*$ The patients should always wear warm clothing.

Chloroform has lately been used by the French. The usnal practice of Dr. Aran is, to apply a compress dipped in chloro. form to the abdomen for the space of twenty minutes, and at the same time to give internally about thirty drons in mueilage. These doses are repeated according to circumstances. He has treated eight cases in this manner, all of which recovered in from two to six days, (see Provincial Journal for March, 1851.) I think chloroform may probably be used with advantage, and may diminish pain in the acute species, and be useful in the chronic form, and I have no doubt many of the suffering patients will willingly submit to its use in any way.

- $\mathbf{H}-$, aged forty, has been employed as a gliss-cutter the last twenty years; for six weeks has complained of frequent severe griping pain in the bowels, not increased by pressure, with pain in the lower part of the loins, and in most of the muscles of the extremities, with many of the other symptoms before detailed, but not constipation. With these symptoms in a slighter degree he partially followed his occupation several weeks, till he was so worn down by suffering and debility that he was unable to leave the house, and seat for me. I now for the first time examined his gums, and the blue tinge at once pointed out the nature of his complaint. He was soon convalescent by the usual remedies. The pains left the extremities and settled in the abdominal and pectoral muscles.

- C- aged thirty-five, a painter for twenty years, has lately suffered severely, and with the usual symptoms, with blue gums; he also had diarrhoa, vomiting, and strangury, several weeks before he applied to me. Both these patients had two severe attacks within a period of fourteen months, and $\mathrm{C}-$, had an attack of gout in both feet during convalescence.

To show the direct influence lead has on the muscular system, we often see paralysis of the right arm in painters, and more particularly in the adductor pollicis in glaziers, whose frequent occupation is to spread the "putty" with the thumb, to fasten panes of glass in the windows; and putty is a composition of lead.

In the acnte form of the disease I have frequently seen numerous successive paroxysms for months, and I have witnessed a succession of attaclss in the chronic species.

Persons will sometimes be employed in lead manufactories ten or twenty years, and remain free from violent suffering, but rarely free from slight symptoms; and when severe attacks have once occurred, without great care patients may be martyrs to the disease, which may eventually destroy life.
The gums of those patients employed in lead should occasionally be examined, and when they assume a slight blue tinge, proper remedies and precautions should be adopted for I consider no patient safe till the gums wear a healthy colour, and patients will be doomed to fresh and perhaps more severe altacks. The long-continued suffering produces such a depression in the nervous system, that some of the patients appear willing to destroy themselves; and in the latter stages,

"Yellow sickness sits cavern'd in the hollow eye."

I have had an opportunity of rare occurrence of witnessing the deloterious effects ot lead on the human frame, in consequence of a miller's servant, by mistake, mixing thirty pounds of acetate of lead with about eighty sacks of flour, already detailed by me in the Provincial Journal published in June, 1849, nnd in THe Lances for August, 1849; and as the acute or chronic form of the disease mas witnessed by me daily for many months, it induced me to investionte the disease, and to drew the foregoing deductions and conclusions; and I trust and hope that toxicologicts will carry on the inquiry, as the health of thousands may be benefited.

Dr. Watson, in his very valuable lectures, states that Mr. Tones, a dentist, has come to the conclusion that the blue tinge in the gums is produced by some chemical action between the tartar that forns on the teeth where they meet the gums, and the lead which pervades the system; but when the system is saturated with liad, I feel convinced the blue tinge will oconr where no tartar exists; for I lave frequently seen it in young persons, aid also in childreie.

Stourbridge, Worcestershire, 18 šl.

\section{A CASE OF INSANITY CURED BY A SURGICAL OPERATION.}

Br WILLIAM BAYARD, M.D., \&c., St. John's, New Brunswick.

RoRERT T-, of Conchiboguac, in New Brunswick, twentythree years of age, was placed in the Provincial Lunatic Asylum, having suffered for some time under mental derangement. His father stated, in reply to interrogatories, that his right eye was injured at the time of his birth-as he thought, by the midwife-it being much swollen and inflamed, in which state it continued for three months, when it became better, though permanently enlarged; that, about four years ago, the same eye was injured by a blow from a chip, when cutting wood; that the eyelid was cut, but he cannot say whether the eyeball was punctured. There was an occasional discharge from it till about six months prior to the operation, when it ceased, and was followed by an increase in its size, accompanied with frequent pain in the head. His intellect was unimpaired till this period, when he manifested unequivocal symptoms of insanity, and, preferring his bed, he continued in it constantly.

He remained in the lunatic asylum, under the charge of Dr. Waddle, the medical superintendent, for two months, withont experiencing any improvement in his symptoms, when my father, Dr. R. Bayard, and myself, were called upon to visit him: we did so, and found him in bed, muttering a few words in a low and mournful tone of voice. He seemed totally regardless of surrounding objects; there was an expres. sion of pain in his countenance, and he was disinclined to any lind of exertion, though his manner and temper were gentle; he suffered from great want of sleep; the eyeball was enlarged to about the size of a small orange, protruding beyond the orbit, and much disorganized; there was no appearance of pupil or iris, and the eyelids adhered throughout their entire surface to the selerotic membrane. The increased size of the eye was evidently owing to an increased quantity of fluid within its coats, and it was very firm and hard.

The general health of the patient was declining, characterized by loss of flesh and strength, impaired sleep, and constipation of bowels. He took food apparently more as an act of obedience than from any appetite for it. He preferred his bed, but when taken from it his movements were unsteady from debility. The history, progress, and symptoms of the disease of the eye, and the subsequent insanity, induced us to believe that an operation offered the only prospect of relief. The case, with its uncertainties and possibility o: relief, was represented to his father, who imme. diately assented to the performance of the operation; and accordingly, on the $22 n d$ of May, 1850, he was removed from the asylum to St. John's. Previous to operating, the patient was subjected to the iufuence of chloroform, which, although freely exhibited, occasioned only slight ex- 